\title{
Practical safety in the diagnosis and treatment of congenital isolated tracheoesophageal fistula
}

\author{
RADU-IULIAN SPATARU ${ }^{1,2}$, DAN-ALEXANDRU IOZSA ${ }^{1,2}$, MIRCEA OVIDIU DENIS LUPUSORU $^{3}$, \\ DRAGOS SERBAN ${ }^{4,5}$ and CATALIN CIRSTOVEANU ${ }^{6,7}$
}

\begin{abstract}
${ }^{1}$ Discipline of Pediatric Surgery, Department of Plastic and Reconstructive Surgery and Pediatric Surgery, Faculty of Medicine, 'Carol Davila' University of Medicine and Pharmacy, 020021 Bucharest; ${ }^{2}$ Department of Pediatric Surgery, Emergency Clinical Hospital for Children 'Maria Sklodowska Curie', 41451 Bucharest; ${ }^{3}$ Discipline of Physiology, Department 2, Faculty of Medicine, 'Carol Davila' University of Medicine and Pharmacy; ${ }^{4}$ Discipline of General Surgery, Department of General Surgery, Faculty of Medicine, 'Carol Davila' University of Medicine and Pharmacy, 020021 Bucharest; ${ }^{5}$ Department of General Surgery, Emergency University Hospital, 050098 Bucharest;

${ }^{6}$ Discipline of Pediatrics, Department of Pediatrics, Faculty of Medicine, 'Carol Davila' University of Medicine and Pharmacy, 020021 Bucharest; ${ }^{7}$ Department of Neonatal Intensive Care Unit, 'Marie S. Curie' Emergency Clinic Hospital for Children, 41451 Bucharest, Romania
\end{abstract}

Received January 22, 2021; Accepted February 22, 2021

DOI: $10.3892 / \mathrm{etm} .2021 .9970$

\begin{abstract}
The rareness of H-type tracheoesophageal fistula in conjunction with its unspecific clinical presentation and wide range of anatomical presentation makes its diagnosis and treatment a problematic topic for both ear, nose and throat (ENT) specialists and pediatric surgeons worldwide. Symptoms and clinical signs of H-TOF are easily misleading. Diagnostic methods, most of the times, are dependent on the physician's experience; therefore, various errors may be made. We analyzed our experience in managing H-TOF cases over the last 15 years. Advice and strategies of action for health professionals directly involved in the diagnosis and treatment were identified, but also errors and mistakes while managing 6 cases. We analyzed 'red flags' but also important steps in the practical safety concerning this rare congenital malformation. Choosing the surgical access for division of the fistula throughout the cervical or thoracic approach is sometimes difficult. A scrupulous perioperative planning is mandatory. A dynamic overview of the patient's presentation never
\end{abstract}

Correspondence to: Dr Dan-Alexandru Iozsa, Discipline of Pediatric Surgery, Department of Plastic and Reconstructive Surgery and Pediatric Surgery, Faculty of Medicine, 'Carol Davila' University of Medicine and Pharmacy, 37 Dionisie Lupu Street, 020021 Bucharest, Romania

E-mail: dan.iozsa@yahoo.com

Abbreviations: H-TOF, congenital H-type (isolated) tracheoesophageal fistula; GERD, gastro-esophageal reflux disease

Key words: isolated tracheoesophageal fistula, esophageal atresia, $\mathrm{H}$-type tracheoesophageal fistula, practical safety, gastro-esophageal reflux disease underestimating the subtlety of H-TOF presentation should be conducted for its early recognition and achieving best outcomes.

\section{Introduction}

Isolated tracheoesophageal fistula (or H-type tracheoesophageal fistula) is a rare congenital disease described for the first time in a 7-week-old infant by Lamb in 1873 (1). It is one of the main five anatomical types of congenital esophageal malformations, as it appears in both Vogt and Gross classifications, which are still the most commonly used today (2). Medical literature shows that both ear, nose and throat (ENT) specialists and pediatric surgeons are involved in $\mathrm{H}-\mathrm{TOF}$ management.

H-TOF accounts for less than 5\% of all congenital tracheoesophageal malformations (3). The most common presentation includes choking and coughing during feeding, repeated episodes of pneumopathy, recurring cyanosis and abdominal distension (4). Even though these are recognized early in the first days of life, the final diagnosis is often delayed. This is a consequence of the broad spectrum of differential diagnosis in infancy, taking into account more frequent neonatal pathology such as perinatal asphyxia, with similarities in clinical presentation, the rareness of the anomaly and, moreover, the low sensitivity and specificity of the available evaluation tools whose results are also dependent on the experience of the health professional, mainly the surgeon or the radiologist. Often, repeated evaluations are reported to be required until $\mathrm{H}-\mathrm{TOF}$ is recognized since it is difficult to diagnose after a single test $(4,5)$.

Imperatori reported the first repair of a H-TOF in 1939 in a 6-year-old child via a trans-tracheal route. Haight, reported in 1948 the first thoracic approach in H-TOF repair and Miller, the first trans-cervical division of an H-TOF located close to 
the thyroid $(1,3)$. The main goal in the surgical treatment of $\mathrm{H}-\mathrm{TOF}$, is division of the fistula. This may be achieved through a thoracic or cervical access with simple ligation or excision of the fistula tract followed by tracheal and esophageal repair (3). Fistula identification, with a clear preoperative and intraoperative picture over the fistula's position and-therefore, choosing the right approach-is a centerpiece of H-TOF management. The aim in surgical planning is to avoid extensive and unnecessary dissection and thus potential complications taking into account the hazardous anatomy of the cervical region or the posterior mediastinum, especially in newborns or infants $(4,6)$.

\section{Patients and methods}

Six patients were treated for H-TOF at the Pediatric Surgery Clinic, Emergency Clinical Hospital for Children 'Maria Sklodowska Curie', Bucharest, Romania, between 2005 and 2019. Informed consent was obtained from the patient's parents. The medical records of these patients were analyzed and the following information was summarized into short case series: history and clinical presentation, preoperative evaluation tools and observations, age at surgery, surgical approach and intraoperative comments. By compiling our data and correlating it to the current knowledge, we wish to highlight 'error traps' or challenges that we have encountered and other possible 'red flags' and establish a relevant view of the practical safety in the diagnosis and management of this rare congenital malformation.

\section{Results}

The sex distribution of our series describes 4 male and 2 female patients. The youngest patient was diagnosed at 15 days of life, while surgery was performed at 38 months in the oldest one. The median age of our series was 19 months and the mean age was 12 months. Feeding difficulties (choking, especially during liquid swallowing) and functional respiratory syndrome were the most frequently noted clinical aspects. One case presented with associated tracheomalacia. No other associated congenital malformations were noted in this series. Regarding other comorbidities, failure to thrive was noted in two cases and two cases of associated gastroesophageal reflux disease (GERD). We also report one case of H-TOF with matrilineal heredity with a 2nd degree relative (mother's sister) with history of esophageal atresia and distal tracheoesophageal fistula. Esophagogram was performed in all cases with a true positive rate of $66 \%$ (4 out of 6). Esophagoscopy was conducted in three cases, indicating fistula in only one case, while tracheobronchoscopy was performed in three cases identifying the fistula in each of them. The fistula was approached through open right thoracotomy in three cases and via cervicotomy in the other three (7).

Case report 1. A 38-month-old female patient was referred to the pediatric surgery department for occasional choking during feeding, especially liquid foods. History revealed periodic episodes of pneumopathies with repeated admissions for special care. The performed esophagogram revealed an H-TOF of T3-T4 level which was further repaired by right open thoracic access.
Case report 2. An 18-month-old male infant was referred for feeding difficulties (choking during meal times) and associated cyanosis. No relevant history was noted excepting occasional cough paroxysms, ignored by the family. Esophagogram was performed showing a C7-T1 level H-TOF which was operated via right cervicotomy.

Case report 3. A 3-month-old male infant presented to the emergency room for severe coughing and choking which worsened at feeding attempts leading to cyanosis. Esophagogram was inconclusive. T2-T3 H-TOF fistula was confirmed by esophagoscopy at $11 \mathrm{~cm}$ from the incisor line. Identification was possible after the anesthesiologist was asked to assist by hyperventilation of the patients so that air bubbles could be identified in the esophagus. Right thoracotomy was performed in order to ligate and divide the fistula.

Case report 4. A 2-month-old male infant was transferred to our hospital for constant coughing and choking precipitated by feeding attempts. Clinical examination revealed functional respiratory syndrome and poor weight gain $(200 \mathrm{~g}$ since birth). Previous history showed a prolonged hospitalization since birth for neonatal sepsis associated with functional respiratory syndrome and choking at feeding attempts for which neurological swallowing disorder was considered and nasogastric tube feeding was indicated. Fluoroscopic swallowing exam and esophagogram were conducted shortly after admission to our clinic showing a T3 level H-TOF (Fig. 1) and invasion of barium solution into trachea and larynx which was later confirmed by tracheobronchoscopy (Fig. 2). Right thoracic open approach was performed in this case (Fig. 3).

Case report 5. A 14-day-old male newborn was referred to our neonatal intensive care unit for a unique episode of pneumonia shortly after birth. Excessive secretions in the oro-pharynx, dysphonic cry and mild respiratory functional syndrome were noted in regards to the clinical observations. Esophagogram was performed with a false-negative result. Therefore, tracheobronchoscopy was performed revealing the H-TOF $2 \mathrm{~cm}$ below the larynx and tracheomalacia. Left cervicotomy access permitted ligation and division of the fistula under safe conditions (Fig. 4).

Case report 6. A 10-month-old infant was admitted for medical care for aspiration pneumonia. Recurrent episodes of upper respiratory tract infections and GERD were outlined in the patient's history. No other associated anomalies were noted. Clinical checkup highlighted poor weight gain (4,500 g since birth). Esophagogram imagery was achieved and high GER was described. Upper endoscopic assessment did not reveal any signs of esophagitis. Therefore, the patient underwent Nissen fundoplication surgery considering the high-grade gastro-esophageal reflux complicated by repeated aspirations. The patient had a poor outcome in the early postoperative period in regards to respiratory function recovery and therefore tracheobronchoscopy was performed revealing H-TOF which was repaired using a right cervical approach. 


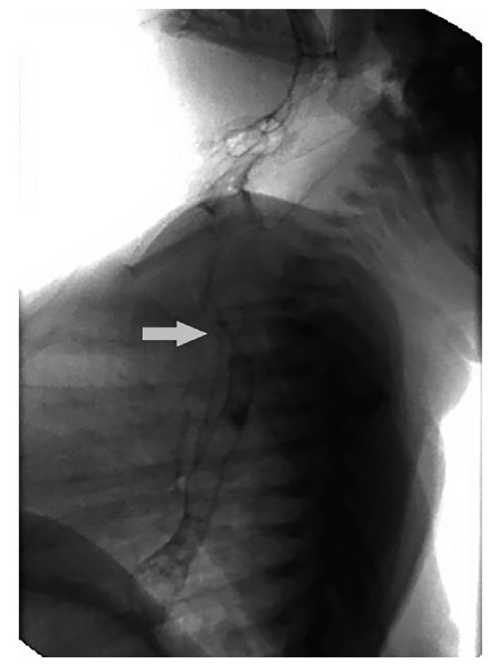

Figure 1. Esophagogram showing T3 level H-TOF. H-TOF, congenital H-type (isolated) tracheoesophageal fistula.

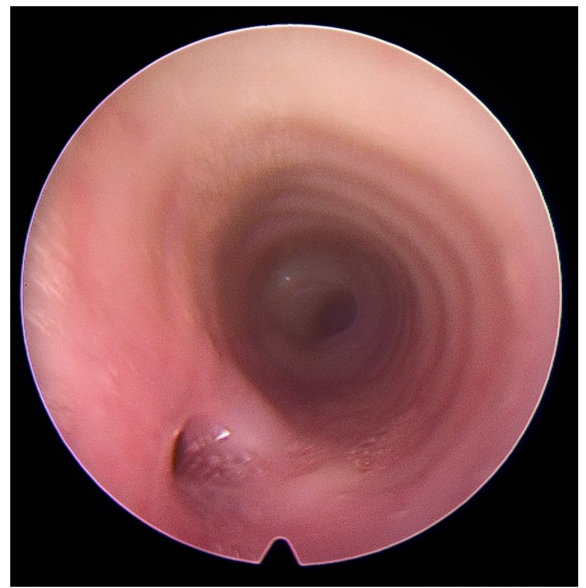

Figure 2. Bronchoscopic aspect of H-TOF. H-TOF, congenital H-type (isolated) tracheoesophageal fistula.

\section{Discussion}

Generally, the treatment of rare pathological conditions in the pediatric population is concentrated in highly-specialized tertiary centers. The expertise of the health professionals in these centers is an important factor for a better outcome of these patients.

Congenital H-type (isolated) tracheoesophageal fistula (H-TO) has an incidence of about 1:50,000-100,000 individuals and a slightly higher prevalence in males (3). It is sporadically reported in the current literature in small series (8). Almost all patients are diagnosed within the first three years of life, most of the cases being recognized before the age of 12 months, respectively in the neonatal period $(4,9)$, while $\mathrm{H}$-type TOF is rarely diagnosed beyond childhood and even more limited in adult surgical experience $(10,11)$.

The clinical key points for H-TOF diagnosis are classical and they are represented by coughing or paroxysmal choking during feeding, pneumopathy or history of pneumopathy, repeated cyanosis and, infrequently, abdominal distension $(4,5)$, which are often intermittent and they are related to fistula size,

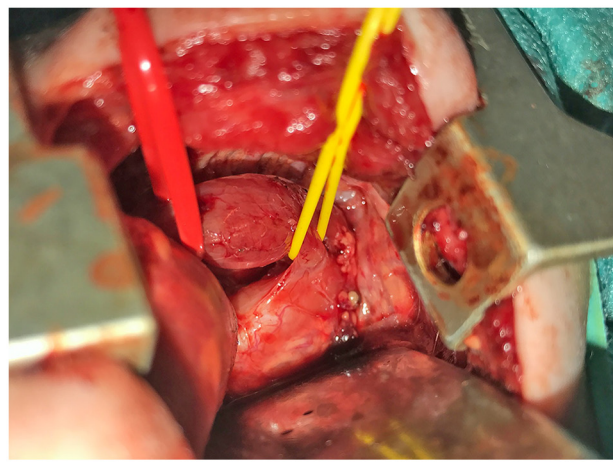

Figure 3. Intraoperative aspect of H-TOF, thoracic approach. H-TOF, congenital H-type (isolated) tracheoesophageal fistula.

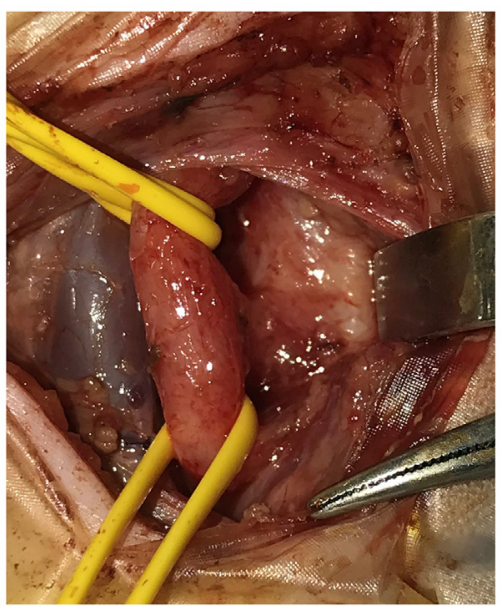

Figure 4. Intraoperative aspect of H-TOF, cervical approach. H-TOF, congenital H-type (isolated) tracheoesophageal fistula.

co-existence of respiratory effort or endotracheal intubation anesthesia $(3,9)$.

Isolated tracheoesophageal fistulas are a very rare group of congenital malformations. Continuity of esophagus pictures H-TOF as an exclusive presentation over the anatomical variants of congenital tracheoesophageal anomalies met in the clinical practice $(5,12)$. A noticeable fraction of H-TOF patients (19-50\%) present with other malformations or comorbidities such as cardiac defects, anorectal malformations, cleft lip, laryngeal cleft or may present multiple malformations such as VACTERL associations, CHARGE syndrome, Goldenhar syndrome, Down or Opitz syndrome (13-15). Therefore, the symptoms may be easily misleading or diverted when an accurate workup plan is not conducted $(4,14)$. All of these may act as a 'smokescreen' in the diagnostic pathway. While Fallon et al identified 29\% of H-TOF cases as preterm (8), some authors advocate prematurity-related conditions (such as respiratory distress) motivating the delay in diagnosis (15). We would like to strengthen this idea by adding the importance of neonatal dysphagia and swallowing disorders which has an incidence of $13 \%$ in the general population which is doubled (26\%) in preterm babies (16).

A meaningful misleading element in the early diagnosis of H-TOF is gastroesophageal reflux disease (GERD). The extensive set of symptoms and complications that GERD 
may present (both gastrointestinal-regurgitation, vomiting, dysphagia, failure to thrive, or regarding the airways-wheezing, stridor, recurrent pneumopathy) (17) may easily lead to clinical judgement bias considering the rarity of H-TOF. More than that, the diagnosis of H-TOF may be missed because of the low specificity of esophagogram and esophagoscopy and furthermore, of the masquerading symptoms if nasogastric tube feeding is initiated (5). Fallon et al reports, in his multicentric review (8), three cases of fundoplication diagnosed prior to $\mathrm{H}-\mathrm{TOF}$ repair suggesting a pitfall in fistula diagnosis. Within our small period of experience, one case out of six presented the same pattern. GERD is alleged by Tarcan et al (15) in his isolated case for its confounding symptoms in preterm babies. In addition, taking previous statements into account and the predilection site of H-TOF above the T2 vertebra level (4), gastroesophageal reflux identified on esophagogram, especially grade II-III or higher according to McCauley's classification (18), has a high probability to generate unexplained recurrent $\mathrm{H}-\mathrm{TOF}$ symptoms (in the absence of feeding) as a consequence of the gastroesotracheal reflux.

Work-up on any suspicion of H-TOF should begin with a simple plain chest X-ray and this is usually performed considering the practical generally agreed consensus upon the initial evaluation of respiratory symptoms. This exam is not helpful, but signs such as gaseous distension of the gastro-intestinal tract and especially of the esophagus are elusive $(4,5)$ and might be easily missed. Taking these facts into account, we would like to point out an article by Boybeyi et al depicting 5 cases of megaesophagus on chest X-ray indicating H-TOF in patients diagnosed late (between 12 and 22 years of age), invoking caution and the role of manometry before deciding to perform an esophagomyotomy since the chronic effects of H-TOF may be complicated (19). Thus, it is important to raise a 'red flag' for any patient beyond childhood with unexplained esophageal dilatation with an associated history related to the H-TOF clinical picture. Therefore, an esophagogram exam in a prone or supine position is usually recommended. This is considered by many authors sufficient for confirmation of the diagnosis and beneficial for the exclusion of other diagnoses such as cleft larynx, gastroesophageal reflux, swallowing discoordination (5). Yet, this may be accompanied by a series of pitfalls, with the sensitivity ranging from 50 to $73 \%$ in H-TOF (20). A series of arguments may be taken into account: a) the ' $N$ ' aspect of the fistula in which the esophageal opening is lower than the tracheal orifice creating a tight space between the two lumens and causing fistula to clog most of the time; b) normal active swallowing movements may not be strong enough to generate sufficient esophageal distension and consecutive contrast substance invasion to the trachea and the contrast agent may need to be injected under pressure; c) esophageal mucosal folds or esophageal muscular spasm which may keep the digestive opening of the fistula closed. The contrast substance may be transient in the respiratory tree during the examination, coughing being able to eliminate it. For this reason, radioscopy or continuous acquisition of films with image amplification are indicated to boost the chances of fistula identification. On the other hand, considering that the contrast agent should be instilled up in the cervical region to delineate the esophagus as much as possible and not to miss high fistulas, laryngeal aspiration of the contrast agent is more possible. This may be produced either directly through the H-TOF if positive, or in the context of high GERD. In the later eventuality, the contrast agent can be present in the respiratory tree without a visible fistula path if the radiologist is not dynamically achieving imagery. Accordingly, a false-negative esophagogram should be considered as long as clinical doubt persists. Radiological studies in the premise of $\mathrm{H}-\mathrm{TOF}$ should be made with particular vigilance in sites with quick access to intensive care units and professionals prepared for prompt resuscitation and, preferably, by experienced radiologists. Isotonic water-soluble contrast agents are recommended $(5,9,13)$. In our experience, barium swallow has been used to obtain conclusive images of H-TOF and the preparation of a low viscosity solution (dilution) plays a significant role in obtaining adequate images of the tracheoesophageal passage. This may lead to a more facile aspiration or to lesser enhanced contrast pictures, and we support the rapid obtaining of many frames during pharyngeal and esophageal stages of deglutition.

Esophagoscopy is less useful in H-TOF identification since the esophageal ostium is smaller and the intraluminal air-pressure during the digestive endoscopy may intermittently close the opening with mucosal folds $(3,5,13)$.

Tracheobronchoscopy represents the most reliable tool in regards to clinical tracheoesophageal fistula suspicion $(4,20)$. This test can also discern other associated malformations such as laryngeal cleft, laryngeal stenosis or tracheomalacia (20). In contrast with the esophagus, the firm aspect of the trachea makes the tracheal opening of the fistula easier to be visualized. In the case of doubt or difficulties in visualization a series of applicable guidelines are suggested in previous studies: i) Passing a suction catheter tip over the posterior tracheal wall may drop into a blind fistula opening (3); or ii) more complex mixed endoscopic technique such as injecting saline solution into the esophagus along with positive pressure ventilation may produce air bubbles at the fistula site (4) or esophageal instillation with methylene blue (6). In addition to its diagnostic value, tracheobronchoscopy is recommended preoperatively or during surgery in order to localize the fistula and to decide the best approach, avoiding long, meaningless, hazardous dissections. In this order, a Fogarty catheter may be inserted through the tracheal ostium, but this may migrate during surgical or anesthetic procedures. Garcia et al (6) and Ko et al (21) introduced the idea of knot locked guide wire passed through the fistula before orotracheal intubation using both esophagoscopy and bronchoscopy. Recently, Goyal et al (22) promoted the idea of using intraoperative transillumination of the fistula using flexible miniature bronchoscopy. Nevertheless, the value of tracheobronchoscopy in order to exclude extraordinary cases of more than one congenital tracheoesophageal fistula without esophageal atresia in the same patient should not be ignored $(23,24)$.

Other diagnostic and preoperative evaluation tools including virtual bronchoscopy and direct sagittal CT scan have been reported in the current literature, but their benefits are limited (5).

The first milestone in H-TOF repair is providing clear landmarks of its level. Most frequently, the fistula is located at the thoracic inlet having an oblique course with the esophageal ostium opening lower. Cervical approach is recommended for fistulas above the T2 vertebra level and thoracic approach is 
the procedure of choice in cases where the fistula is below this level (3). Regarding the side of the cervical incision, right-side is preferred by most pediatric surgeons who claim a reduced risk of recurrent laryngeal nerve damage which arises more superiorly on this flank. In contrast, ENT surgeons, more involved in high fistulas, prefer the left side for a better access over the cervical esophagus. Right-side is also preferred for low cervical H-TOF presentations $(4,25)$. Concerning the thoracic approach, we prefer open right-side surgery because of the position of the aorta, and the better access to the vagus nerve on this flank. It is important to consider malposition of the aortic arch to the right prior surgery. Moreover, in case of high thoracic fistulas, cautious dissection should be conducted keeping in mind the position of the sympathetic plexus which once injured may cause unwanted disorders such as Horner's syndrome. From our experience, when surgical access gets difficult, head and neck repositioning of the patient may have an important role relatively changing the fistula's position, keeping in mind a potential endotracheal tube displacement. Minimally invasive access has cosmetic and magnification advantages, does not seem to be less beneficial in regards to postoperative morbidity and does not present with worse outcomes than the open approach in children. Yet, this procedure is complex and should be taken into consideration by well-trained surgeons in properly equipped facilities (20,25). Endoscopic approach using electrocautery, laser or tissue glue to obliterate the fistula have been attempted with arguable results $(3,24)$; therefore these techniques are not promoted by us.

The second milestone of H-TOF surgery is its intraoperative identification. In extremely rare cases, but possible as previously reported, the fistula may be associated with a duplication cyst which may enable difficulty in its identification and dissection (26). We discussed this above along with the advantages of tracheobronchoscopy. In our experience, we did not find any major difficulties in establishing the fistula site. Asking the anesthesiologist to induce positive tracheal pressure by disconnecting the patient from mechanical ventilation and using rebreathing bag for a while or by injecting air through a nasogastric tube located in the esophagus was very useful.

The last event in H-TOF repair is division and ligation of the fistula using interrupted sutures on both tracheal and esophageal sides of it. Transection should be performed sharp on the tracheal side in order to prevent diverticula formation taking into consideration our overall experience in esophageal atresia. We support the suggestions of other authors (11) to position a muscular flap between the trachea and esophagus or keeping a small cuff of peritracheal connective tissue to ensure the tracheal suture. It is considered that these procedures will promote better healing and will prevent leakage even though its effectiveness is not clearly demonstrated. We also favor the use of a drain tube and we recommend Penrose-type since it does not clog.

Postoperative complications in $\mathrm{H}-\mathrm{TOF}$ are proven to be precipitated by the coexistence of other comorbidities. Reported events are suture dehiscence, edema, fistula recurrence, stridor/dysphonia or vocal cord palsy/paresis, esophageal stenosis, swallowing difficulties, prolonged intubation or pulmonary distress. Vocal cord palsy/paresis seem to be related to technical issues, while suture permeability complications seem to be more prevalent in children with pre-existing GERD. Therefore, proper pharmacological control is recommended preoperatively and nevertheless, bearing in mind that GERD and esophageal dysmotility are also known as postoperative complications. Extubating should be carefully conducted in order to prevent complications or reintubation by taking into account edema and associated conditions $(3,4)$.

In conclusion, H-TOF management is not easy, therefore it must be dedicated to experienced and skillful surgeons who should utilize a well-experienced team in an adequately technically supplied facility. The diagnosis should not be easily excluded (especially in newborns and infants) when respiratory symptoms persist. Revision of previously performed work-up should be reconsidered with a consonant tenacity including meticulous identification of associated conditions. Moreover, the surgeon should keep in mind that the H-TOF diagnosis process is often crucial to 'how' the evaluation tools are used. A special place in diagnosis and treatment of H-TOF is gastroesophageal reflux which may be a significant factor of confusion or act as a booster for postoperative comorbidities. Cautious preoperative preparation, fully aware of the anatomical landmarks and dissection steps that will be encountered, builds up the best strategy and leads to favorable outcomes.

\section{Acknowledgements}

Not applicable.

\section{Funding}

No funding was received.

\section{Availability of data and materials}

All data generated or analyzed during this study are available in this published article.

\section{Authors' contributions}

RIS, DAI and CC contributed directly to the patient diagnoses, surgical and intensive care unit management. RIS and DAI designed, conceived and wrote the manuscript. DS and MODL reviewed the literature and edited the article. All authors approved the final manuscript.

\section{Ethics approval and consent to participate}

The study was approved by the Ethics Committee of the 'Marie S. Curie' Emergency Clinic Hospital for Children having the hospital registry number 38689/21.10.2020. All parents signed an informed consent, allowing the use of all medical data for academic purposes, including possible scientific publication of them, respecting their anonymous character.

\section{Patient consent for publication}

Not applicable.

\section{Competing interests}

The authors declare that they have no competing interests. 


\section{References}

1. Lacquet A and Fransen G: Isolated Tracheoesophageal Fistula In: Diseases of the Esophagus. Handbuch der Inneren Medizin (Verdauungsorgane). Vol 3. Springer, Berlin, pp639-642, 1974.

2. Spitz L: Oesophageal atresia. Orphanet J Rare Dis 2: 24, 2007.

3. Al-Salem AH, Mohaidly MA, Al-Buainain HM, Al-jadaan S and Raboei E: Congenital H- tracheoesophageal fistula: A national multicenter study. Pediatr Surg Int 32: 487-491, 2016.

4. Genty E, Attal P, Nicollas R, Roger G, Triglia JM, Garabedian EN and Bobin S: Congenital tracheoesophageal fistula without esophageal atresia. Int J Pediatr Otorhinolaryngol 48: 231-238, 1999.

5. Ng J, Antao B, Bartram J, Raghavan A and Shawis R: Diagnostic difficulties in the management of H-type tracheoesophageal fistula. Acta Radiol 47: 801-805, 2006.

6. Garcia NM, Thompson JW and Shaul DB: Definitive localization of isolated tracheoesophageal fistula using bronchoscopy and esophagoscopy for guide wire placement. J Ped Surg 33 1645-1647, 1998.

7. Bratu N, Spătaru RI and Iozsa DA: Isolated tracheoesophageal fistula-a rare congenital malformation. J Pediatr 17: 67-78, 2014.

8. Fallon SC, Langer JC, Peter SD, Tsao K, Kellagher CM, Lal DR, Whitehouse JS, Diesen DL, Rollins MD, Pontarelli E, et al: Congenital H-type tracheoesophageal fistula: A multicenter review of outcomes in a rare disease. J Ped Surg 52: 1711-1714, 2017.

9. Gardella C, Toma P, Sacco O, Girosi D, Panigada S, Battistini E, Mattioli G, Jasonni V and Rossi GA: Intermittent gaseous bowel distension: Atypical sign of congenital tracheoesophageal fistula Pediatr Pulmonol 44: 244-248, 2009.

10. Hajjar WM, Iftikhar A, Al Nassar SA and Rahal SM: Congenital tracheoesophageal fistula: A rare and late presentation in adult patient. Ann Thorac Med 7: 48-50, 2012.

11. Suen HC: Congenital H-type tracheoesophageal fistula in adults. J Thorac Dis 10 (Suppl 16): S1905-S1910, 2018.

12. Riazulhaq $M$ and Elhassan E: Early recognition of H-type tracheoesophageal fistula. APSP J Case Rep 3: 4, 2012.

13. Crabbe DC, Kiely EM, Drake DP and Spitz L: Management of the isolated congenital tracheo-oesophageal fistula. Eur J Pediatr Surg 6: 67-69, 1996.

14. Fraga JC, Adil EA, Kacprowicz A, Skinner ML, Jennings R, Lillehei $\mathrm{C}$ and Rahbar R: The association between laryngeal cleft and tracheoesophageal fistula: Myth or reality? Laryngoscope 125: 469-474, 2015.
15. Tarcan A, Gurakan B, Arda S and Boybat F: Congenital H-type fistula: Delayed diagnosis in a preterm infant. J Matern Fetal Neonatal Med 13: 279-281, 2003.

16. Mercado-Deane MG, Burton EM, Harlow SA, Glover AS, Deane DA, Guill MF and Hudson V: Swallowing dysfunction in infants less than 1 year of age. Pediatr Radiol 31: 423-428, 2001.

17. Rosen R, Vandenplas Y, Singendonk M, Cabana M, DiLorenzo C, Gottrand F, Gupta S, Langendam M, Staiano A, Thapar N, et al: Pediatric gastroesophageal reflux clinical practice guidelines: Joint recommendations of the north american society for pediatric gastroenterology, hepatology, and nutrition and the european society for pediatric gastroenterology, hepatology, and nutrition. J Pediatr Gastroenterol Nutr 66: 516-554, 2018.

18. McCauley RG, Darling DB, Leonidas JC and Schwartz AM: Gastroesophageal reflux in infants and children: A useful classification and reliable physiologic technique for its demonstration. AJR Am J Roentgenol 130: 47-50, 1978.

19. Boybeyi O, Kose M and Ersoz DD: Achalasia-like findings in a case with delayed diagnosis of $\mathrm{H}$-type tracheoesophageal fistula. Pediatr Surg Int 24: 965-969, 2008.

20. Cuestas G, Rodriguez V, Millan C, Munzon PB and Munzon GB: $\mathrm{H}$-type tracheoesophageal fistula in the neonatal period: Difficulties in diagnosis and different treatment approaches. A case series. Arch Argent Pediatr 118: 56-60, 2020.

21. Ko BA, Frederic R, DiTirro PA, Glatleider PA and Applebaum H: Simplified access for division of the low cervical/high thoracic H-type tracheoesophageal fistula. J Ped Surg 35: 1621-1622, 2000.

22. Goyal A, Potter F and Losty PD: Transillumination of H-type tracheoesophageal fistula using flexible miniature bronchoscopy: An innovative technique for operative localization. J Ped Surg 40: e33-e34, 2005

23. Mattei P: Double H-type tracheoesophageal fistulas identified and repaired in 1 operation. J Ped Surg 47: e11-e13, 2012.

24. Sim J and Hong J: Double H-type tracheoesophageal fistulae: A case report. Adv Pediatr Surg 24: 94-99, 2018.

25. Parolini F, Morandi A, Macchni F, Gentilino V, Zanini A and Leva E: Cervical/thoracotomic/thoracoscopic approaches for H-type congenital tracheo-esophageal fistula: A systematic review. Int J Pediatr Otorhinolaryngol 78: 985-989, 2014.

26. Spătaru RI, Popoiu MC and Ivanov M: Foregut duplication cyst associated with esophageal atresia-one-stage neonatal surgical repair. Indian J Surg 77 (Suppl 1): S52-S55, 2014. 